\title{
Time-resolved fluorescence observation of di- tyrosine formation in horseradish peroxidase upon ultrasound treatment leading to enzyme inactivation
}

Konstantina Tsikrika, M. Adília Lemos, Boon-Seang Chu, David H. Bremner and Graham Hungerford

This is the accepted manuscript (C) 2016, Elsevier Licensed under the Creative Commons AttributionNonCommercial-NoDerivatives 4.0 International: http:// creativecommons.org/licenses/by-nc-nd/4.0/

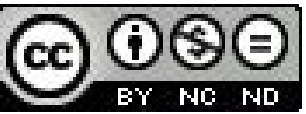

The published article is available from doi:

http://dx.doi.org/10.1016/j.saa.2016.09.035 


\title{
Time-resolved fluorescence observation of di-tyrosine formation in horseradish peroxidase upon ultrasound treatment leading to enzyme inactivation
}

Konstantina Tsikrika ${ }^{a}$, M. Adília Lemos ${ }^{a}$, Boon-Seang Chu ${ }^{a}$, David H. Bremner ${ }^{b}$ and Graham Hungerford $^{c}$

${ }^{a}$ Division of Food \& Drink, School of Science, Engineering and Technology, University of Abertay Dundee, Bell Street, Dundee DD1 1HG, UK.

${ }^{b}$ Division of Science, School of Science, Engineering and Technology, University of Abertay

Dundee, Bell Street, Dundee DD1 1HG, UK.

${ }^{c}$ HORIBA Jobin-Yvon IBH Ltd, 133 Finnieston Street, Glasgow G3 8HB, UK.

Corresponding author G. Hungerford (graham.hungerford@horiba.com)

\begin{abstract}
The application of ultrasound to a solution can induce cavitional phenomena and generate high localised temperatures and pressures. These are dependent of the frequency used and have enabled ultrasound application in areas such as synthetic, green and food chemistry. High frequency $(100 \mathrm{kHz}$ to $1 \mathrm{MHz}$ ) in particular is promising in food chemistry as a means to inactivate enzymes, replacing the need to use periods of high temperature. A plant enzyme, horseradish peroxidase, was studied using time-resolved fluorescence techniques as a means to assess the effect of high frequency ( $378 \mathrm{kHz}$ and $583 \mathrm{kHz}$ ) ultrasound treatment at equivalent acoustic powers. This uncovered the fluorescence emission from a newly formed species, attributed to the formation of di-tyrosine within the horseradish peroxidase structure caused by auto-oxidation, and linked to enzyme inactivation.
\end{abstract}

Keywords- High frequency ultrasound, time-resolved emission spectrum, decay associated spectra, excitation-emission matrix, metal catalysed oxidation. 


\section{Introduction}

Ultrasound has found application in chemistry[1] for mechanochemical activation[2], the synthesis of nanomaterials[3] and in green chemistry[4]. Ultrasound usually refers to sound waves of frequencies above $20 \mathrm{kHz}$ and can be further categorised[5] as "power ultrasound" (20-100 kHz)[6], "high frequency ultrasound" (100 kHz - $1 \mathrm{MHz}$ ) and "diagnostic ultrasound" (1-500 MHz). Ultrasound causes cavitational phenomena, in particular the formation and implosion of small bubbles, whose size is dependent on the frequency employed[7,8]. Bubble implosion generates high localised pressures and temperatures $\left(>5000^{\circ} \mathrm{C}\right)[1]$. Because of the high energy available, this enables free radical formation in the liquid and in the case of water initially promotes the formation of $\mathrm{OH}^{\bullet}$ and $\mathrm{H}^{*}$ radicals[9], which can then lead to the production of hydrogen peroxide and molecular hydrogen. In food chemistry an application of high frequency ultrasound is to induce enzyme inactivation[9] and until recently this had been seen as the preserve of the "power ultrasound" frequency range. The use of high frequency ultrasound is advantageous as it removes the need for a high temperature treatment (for often prolonged times) with an associated negative impact on nutritional content, flavour and colour. More interestingly there are reports that ultrasound treatment time can even augment enzymatic activity[10]; with effects linked to changes in secondary structure[10,11]. Therefore this is an area requiring further study to ascertain the physical effects of high frequency ultrasound upon enzyme structure.

To assess the influence of high frequency ultrasound two frequencies in the "high frequency" range $(378 \mathrm{kHz}$ and $583 \mathrm{kHz}$ ) at similar acoustic powers were chosen. Using these frequencies it is expected that bubbles would have radii in the order of $5 \mu \mathrm{m}$ or less[8], with the higher frequency producing smaller bubbles. The well studied plant enzyme horseradish peroxidase (HRP)[12] was investigated as it contains five tyrosine ( $Y$ or Tyr) and one tryptophan (W or Trp) residue, whose fluorescence behaviour can be employed to elucidate structural changes[13]. Fluorescence is a common technique by which to study conformational changes in proteins and has previously been used to investigate HRP [14-16]. Principally changes in enzyme structure or conformation affect the fluorescence emission of the Tyr and Trp residues because of distance mediated interactions, such as energy transfer by the Förster mechanism. There is similar interaction between them and the haem group, thus changes in structure can be monitored using the fluorescence emission from these amino acids. Using light at $272 \mathrm{~nm}$ enables both Tyr and Trp moieties to be addressed[13]. At this excitation wavelength the overall fluorescence exhibits a hypsochromic shift compared to using a wavelength (ie $295 \mathrm{~nm}$ ) just to excite the Trp[14] and is related to the Tyr contribution. Although Trp emission is commonly used, Tyr emission is also useful to elucidate changes in protein structure[17]. The Trp fluorescence is usually quenched by the presence of the haem[18], while the Tyr emission can be quenched by the presence of the Trp residue[19]. As this fluorescence quenching is distance dependent, conformational changes in both protein 
structure and the active site of HRP can be deduced. The relative position of these fluorescent amino acids in relation to the catalytic haem pocket is shown in Fig. 1. From basic molecular modelling a range of Tyr-Trp distances in the order of $1.3 \mathrm{~nm}$ to $3.5 \mathrm{~nm}$ were obtained, while Tyr-haem distances were in the range $\sim 0.9 \mathrm{~nm}$ to $2.5 \mathrm{~nm}$. The distance from the Trp to the haem was estimated as $1.3 \mathrm{~nm}$, in keeping with a previous report[16]. This means that Tyr-Trp energy transfer is likely as the reported critical transfer distance (distance at which energy transfer is $50 \%$ efficient) is up to $2 \mathrm{~nm}[20]$. Employing more elucidative time-resolved fluorescence techniques with this enzyme enables the decay associated spectra[21] to be obtained to check the influence of high frequency ultrasound on HRP. The effect of this treatment was evident with the formation of a new fluorescent species, emitting close to 407 $\mathrm{nm}$ with a decay time of $-3.5 \mathrm{~ns}$. The spectral changes seen in the time-resolved measurements were also confirmed in a steady state excitation-emission matrix scan and are attributed to the presence of di-tyrosine. It is likely that this was formed by an auto catalytic reaction involving neighbouring Tyr residues in the presence of the haem and hydrogen peroxide, produced by sonication of the solvent. The spectral measurements are supported via an assessment of residual enzymatic activity.

\section{Experimental}

Ultrasound treatment on horseradish peroxidase (EC 1.11.1.7- from Sigma Aldrich) was carried out on the enzyme at a concentration of $5 \mu \mathrm{g} \mathrm{mL}^{-1}$ in deionised water. This dilution was a requirement in order to assess enzymatic activity. Ultrasound was applied for 60 minutes using a Meinhardt Ultraschlltechnik high frequency sonicator at either $378 \mathrm{kHz}$ (acoustic power $32 \mathrm{~W}$ ) or $583 \mathrm{kHz}$ (acoustic power $34 \mathrm{~W}$ ). During the experiment the temperature was controlled by a cooling jacket connected to a cryostatic bath (Fisher Scientific ISOTEMP Thermostatic) to mediate any thermal influence. At the Wattage and frequency used the maximum temperature observed during sonication would be $40^{\circ} \mathrm{C}$. A control experiment at this temperature without the application of ultrasound did not lead to enzymatic inactivation (unlike that seen with sonication). Also at temperatures up to $40{ }^{\circ} \mathrm{C}$ no significant change in HRP secondary structure would be expected, as previously reported using calorimetry [22]. Enzymatic activity was assessed using the oxidation of guaiacol to tetraguaiacol monitored at $470 \mathrm{~nm}$ on a Shimadzu UV-1650PC over a period of 1 minute. $0.5 \mathrm{~mL}$ of $96 \mathrm{~mm}$ guaiacol was used in the presence of $0.5 \mathrm{~mL}$ of $12 \mathrm{mM}$ hydrogen peroxide, $0.4 \mathrm{~mL}$ of deionised water, $1 \mathrm{~mL}$ of $100 \mathrm{mM}$ potassium phosphate buffer $(\mathrm{pH}$ 6.1 ) to which $0.1 \mathrm{~mL}$ (ie $0.005 \mathrm{mg} \mathrm{mL}^{-1}$ ) of enzyme solution was added.

Time-resolved measurements were performed using a Horiba Scientific DeltaFlex with DeltaDiode excitation (DD-270) at $272 \mathrm{~nm}$. At this excitation wavelength both tyrosine and tryptophan residues would be excited. Decay associated spectra were obtained over the wavelength range $295 \mathrm{~nm}$ to $425 \mathrm{~nm}$, with decay data collected at $5 \mathrm{~nm}$ intervals. These data 
were obtained by measuring the time-resolved fluorescence decay for a fixed time at a particular wavelength, then incrementing the wavelength and repeating the measurement process. The resulting dataset was analysed globally with decays modelled by five common decay times in all cases. This was required in order to obtain a good fit and enabled relative changes to be seen between the untreated and treated samples. Excitation-emission matrices (EEMs) scanning the emission spectra for different excitation wavelengths were recorded using a FluoroLog 3. Auxiliary information was obtained by molecular modelling made using ArgusLab (Plenaria Software) by rendering a structure from the RCSB Protein Data Bank (pdb1gwu).

\section{Results and discussion}

Initially Fluorescence excitation-emission matrix (EEM) scans of the HRP, both untreated and ultrasound treated, were made. The outcome is given in Fig. 2, for the untreated sample plus treatment using ultrasound at $378 \mathrm{kHz}$. Similar data was obtained using $583 \mathrm{kHz}$ ultrasound treatment. The presence of both Rayleigh and Raman scattering features from the solvent are evident in the EEMs, the latter because of the low concentration of enzyme (to facilitate activity measurements). It is clear that ultrasound treatment causes a dramatic change in the wavelength of the principal emission from $-335 \mathrm{~nm}$ to $\sim 407 \mathrm{~nm}$. This wavelength is not normally associated with the native (Tyr and Trp) fluorophores present in HRP. There is also a shift in the excitation wavelength, which combined with the emission is indicative of the formation of a new species.

This phenomenon was further explored by the use of time-resolved spectroscopy. Although not completely optimum for this new species, an excitation wavelength of $272 \mathrm{~nm}$ was chosen as this should also excite the principal fluorescent amino acids present (ie Tyr and Trp) as well as the newly formed species (although not as strongly as if a wavelength of $292 \mathrm{~nm}$ was employed). At $280 \mathrm{~nm}$ (close to our excitation wavelength) the extinction coefficients of Tyr and Trp are $1480 \mathrm{M}^{-1} \mathrm{~cm}^{-1}$ and $5540 \mathrm{M}^{-1} \mathrm{~cm}^{-1}$ respectively[23]. Thus nearly twice as much light should be absorbed by Tyr in comparison to Trp, since it out numbers Trp seven to one. The choice of excitation wavelength should also enable any changes in HRP structure to be inferred. A TRES (time-resolved emission spectrum) measurement was performed on the samples, analysis of which allows decay associated spectra to be obtained. This enables the lifetime to be associated with spectral shapes and is a useful technique by which to separate mixtures of fluorophores. The outcome of this analysis is shown in Fig. 3.

The analysis from the time-resolved fluorescence measurements of HRP showed that in all three of the samples the presence of a spectrum relating to a short-lived component (fixed in the analysis at half a histogram bin width, 13ps, to account for a scattered light component) is clearly resolved and can easily be ascribed to Raman scattering from the solvent. Also present is an ill-defined spectrum associated with a lifetime of $\sim 200$ ps making a minor contribution to 
the overall emission. A similar lifetime component has been reported in APO-HRP, which could be indicative of a partial disappearance of the haem group[14]. The removal of an energy transfer pathway from the Trp[18] can cause the shorter-lived fluorescence lifetime component (of the 3 exponentials present) [24] to increase[14]. However, those studies involved selective excitation of the Trp, while here we also excite Tyr. Another study using $280 \mathrm{~nm}$ excitation has reported two sub nanosecond decays, but longer decays on the nanosecond timescale were out of their time window[25]. In solution both free Trp and Tyr exhibit dominant decay associated spectra with a lifetime of $3.4 \mathrm{~ns}$, with minor contributions of 0.54 ns and $0.98 \mathrm{~ns}$ (Trp and Tyr respectively)[13]. So when considering the untreated sample it is unsurprising that the other decay associated spectra resemble a combination of both their emissions, with a longer-lived (9.47 ns) spectrum solely attributable to Trp. However, it should also be kept in mind that at the excitation wavelength used here that, as well as direct excitation of each amino acid, an enhancement in Trp emission is made via energy transfer[19]. Thus the combined spectra (1.38 ns and 3.46 ns components) may also be indicative of this interaction between these two species.

It is evident from the time-resolved data that ultrasound treatment produces a significant change in the spectral behaviour. Although this is also seen in the EEM's, separation of the individual fluorescing components is not so clear. A longer-lived associated spectrum solely attributable to Trp is still present. However, this is a minor contribution and its lifetime is increased in relation to the untreated sample. It should noted that Trp emission in proteins can be multiexponential in nature and component lifetimes as long as 16ns have been reported[26]. The shorter wavelength emission (associated with an $\sim 1.4$ ns decay) is now more reminiscent of Tyr in shape and more strikingly the emission around $407 \mathrm{~nm}$ is seen (in keeping with the EEM) with an associated decay time of $\sim 3.7$ ns. Differences in the relative Trp and Tyr emissions are also observed and there is a dramatic decrease in the enzymatic activity (residual activity in both cases $-3 \%$ ). Examination of the absorption spectra (see Fig. 4) is indicative of the removal of the haem [15], which would account for the lack of activity.

Previous work using HRP has shown an increase in Trp emission when it is excited directly[18]. Denaturing studies on HRP followed by fluorescence have attributed this to initial changes in secondary structure followed by removal of the haem [16] Here, although there is direct excitation of both Trp and Tyr, we could expect a similar behaviour. The decay associated spectra after ultrasound treatment exhibit clear individual Tyr and Trp emission shapes with an apparent decrease in the relative contribution from the Trp emission, rather than the combined shape present before treatment. This observation is consistent with the fact that conformational change in the HRP structure is increasing Tyr-Trp distances and reducing the contribution of Trp emission obtained via energy transfer. Because of the number of Tyr residues present they would absorb more of the excitation light than the Trp. This coupled with a reduction in energy transfer to the Trp results in a relative increase in their contribution to the 
overall fluorescence in comparison to the Trp emission. This process would seem to have more of an influence than any increase in Trp fluorescence associated with haem removal and may indicate a significant change in the HRP secondary structure. A relative increase in shorter wavelength (Tyr) emission has previously been reported using $280 \mathrm{~nm}$ excitation upon denaturing of HRP using $\mathrm{GdHCl}$, where a two step unfolding process was postulated[15]. In that work loss of secondary structure and haem was followed by a second transition associated with the Trp chain.

Although the lack of the haem and changes in protein structure influences the Tyr and Trp fluorescence behaviour it does not account for the emission around $407 \mathrm{~nm}$. HRP has previously been used to act on tyrosine containing substrates in the presence of hydrogen peroxide to catalyse the formation of di-tyrosine[27]. This species, see Fig. 5, is reported to have a fluorescence emission at 407 / $408 \mathrm{~nm}[27,28]$ and formed via a metal-catalysed oxidation in the presence of $\mathrm{H}_{2} \mathrm{O}_{2}$ [27]. These components can be readily found when ultrasound is applied to tyrosine containing HRP in aqueous solution. The metal is present in the haem moiety and $\mathrm{H}_{2} \mathrm{O}_{2}$ can be formed by the dimerisation of $\mathrm{OH}^{\bullet}$ during bubble implosion caused by the application of ultrasound to the solvent[9]. From their proximity Y-233 and Y-234 could be the origin of the longer wavelength emission. Otherwise an interaction involving $\mathrm{Y}$ 185 or Y-201 could be an option. From molecular modelling it is estimated that these residues are separated by $\sim 1.3 \mathrm{~nm}$. Note that the distance between $\mathrm{Y}-201$ and $\mathrm{Y}-233$ is estimated to be $\sim 1.0 \mathrm{~nm}$. For the non adjacent residues to be the origin this would also imply that a very significant change in the HRP secondary structure had occurred.

In this work it is very apparent that the application of ultrasound treatment has had a detrimental influence on the enzyme. Because the control of temperature during the ultrasound treatment this effect cannot be attributed to thermal denaturing of the enzyme. The fact that time-resolved fluorescence has enabled the identification of changes via the formation of a new fluorescing species provides a means to gain further insights on how ultrasound can affect enzyme behaviour. In this regard the effect of different acoustic powers and treatment times require further investigation.

\section{Summary}

Here we have shown that prolonged (60 minute) exposure to high frequency ultrasound can induce changes within the structure of HRP rendering it inactive. The mechanism involves both loss of secondary structure and the removal of the haem. This coupled with ultrasound enhanced formation of hydrogen peroxide, which can interact with the iron present in the haem in a Fenton-like process, results in the formation of di-tyrosine within the HRP structure. Timeresolved fluorescence spectroscopy, making use of decay associated spectra, was shown to be well suited to distinguish the species present and to isolate the effect of Raman scattering 
from the solvent. In this case it is apparent that the formation of di-tyrosine may be used as an indicator for enzyme inactivation and enables the sensitivity of fluorescence to be employed.

\section{Acknowledgements}

Financial support from The University of Abertay R-LINCS programme is gratefully acknowledged.

\section{References}

[1] K. S. Suslick, Science, 1990, 247, 1439

[2] G. Cravotto, E. C. Gaudino and P. Cintas, Chem. Soc. Rev., 2013, 42, 7521.

[3] H. Xu, B. W. Zeiger and K. S. Suslick, Chem. Soc. Rev., 2013, 42, 2555.

[4] M. Sillanpää, T.-D. Pham and R. A. Shrestha, Ultrasound Technology in green chemistry, 2011, pp1.

[5] T. Y. Wu, N. Guo, C. Y. Teh and J. X. W. Hay, Advances in Ultrasound Technology for Environmental Remediation, 2013, pp5.

[6] T. Leong, M. Ashokkumar and S. Kentish, Acoust. Austr.,2011, 39, 54

[7] S. Merouani, O. Hamdaoui, Y. Rezgui and M. Guemini, Ultrason. Sonochem., 2013, 20, 815.

[8] A. Brotchie, F. Grieser and M. Ashokkumar, Phys. Rev. Lett., 2009, 102, 084302.

[9] M. D. N. Islam, M. Zhang and B. Adhikari, Food Rev. Int., 2014, 30, 1.

[10] H. Ma, L. Huang, J. Jia, R. He and L. Luo, Ultrason. Sonochem., 2011, 18, 419.

[11] J. Bhasarker, A. J. Borah, P. Goswami and V. S. Moholkar, Bioresource Technol., 2015, 196, 88

[12] N. C. Veitch, Phytochem., 2004, 65, 249.

[13] J. R. Lakowicz, Principles in Fluorescence Spectroscopy, $3^{\text {rd }}$ Ed, pp529.

[14] T. K. Das and S. Mazumdar, J. Phys. Chem., 1995, 99, 13283.

[15] G. Tsaprailis, D. W. S. Chan and A. M. English, Biochem., 1998, 37, 2004.

[16] H. S. Pappa and A. E. G. Cass, Eur. J. Biochem., 1993, 212, 227.

[17] N. G. Zhdanova, E. A. Shirshin, E. G. Maksimov, I. M. Panchishin, A. M. Saletsky and V. V. Fadeev, Photochem. Photobiol. Sci., 2015, 14, 897.

[18] P. -I. Ohlsson, T. Horre, J. M. Vanderkooi and K. -G. Paul, Acta Chem. Scandinavica, 1986, B40, 257.

[19] M. Lasagna, E. Gratton, D. M. Jameson and J. E. Brunet, Biochem. J., 1999, 76, 443.

[20] M. J. Kronman and L. G. Holmes, Photochem. Photobiol., 1971, 14, 113.

[21] L. Davenport, J.R. Knutson and L. Brand, Biochem., 1986, 25, 1186.

[22] D. G. Pina, A. V. Shnyrova, F. Gavilanes, A. Rodríguez, F. Leal, M. G. Roig, I. Y. Sakharov, G. G. Zhadan, E. Villar and V. L. Shnyrov, Eur. J. Biochem., 2001, 268, 120.

[23] H. Mach, C. R. Middaugh and R. V. Lewis, Anal. Biochem., 1992, 200, 74.

[24] T. K. Das and S. Mazumdar, Eur. J. Biochem., 1995, 227, 823. 
[25] M. T. Neves-Petersen, S. Klitgaard, A. S. L. Carvalho, S. B. Petersen, M. R. A. de Barros and E. Pinho e Melo, Biophys. J., 2007, 92, 2016.

[26] J. A. Schauerte and A. Gafni, Biochem., 1989, 28, 3948.

[27] B. Tang, Y. Wang, H. Liang, Z. Chen, X. He and H. Shen, Spectrochim. Acta A, 2006, 83, 609.

[28] T. G. Huggins, M. C. Wells-Knecht, N. A. Detorie, J. W. Baynes and S. R. Thorpe, J. Biol. Chem., 1993, 288, 12341. 


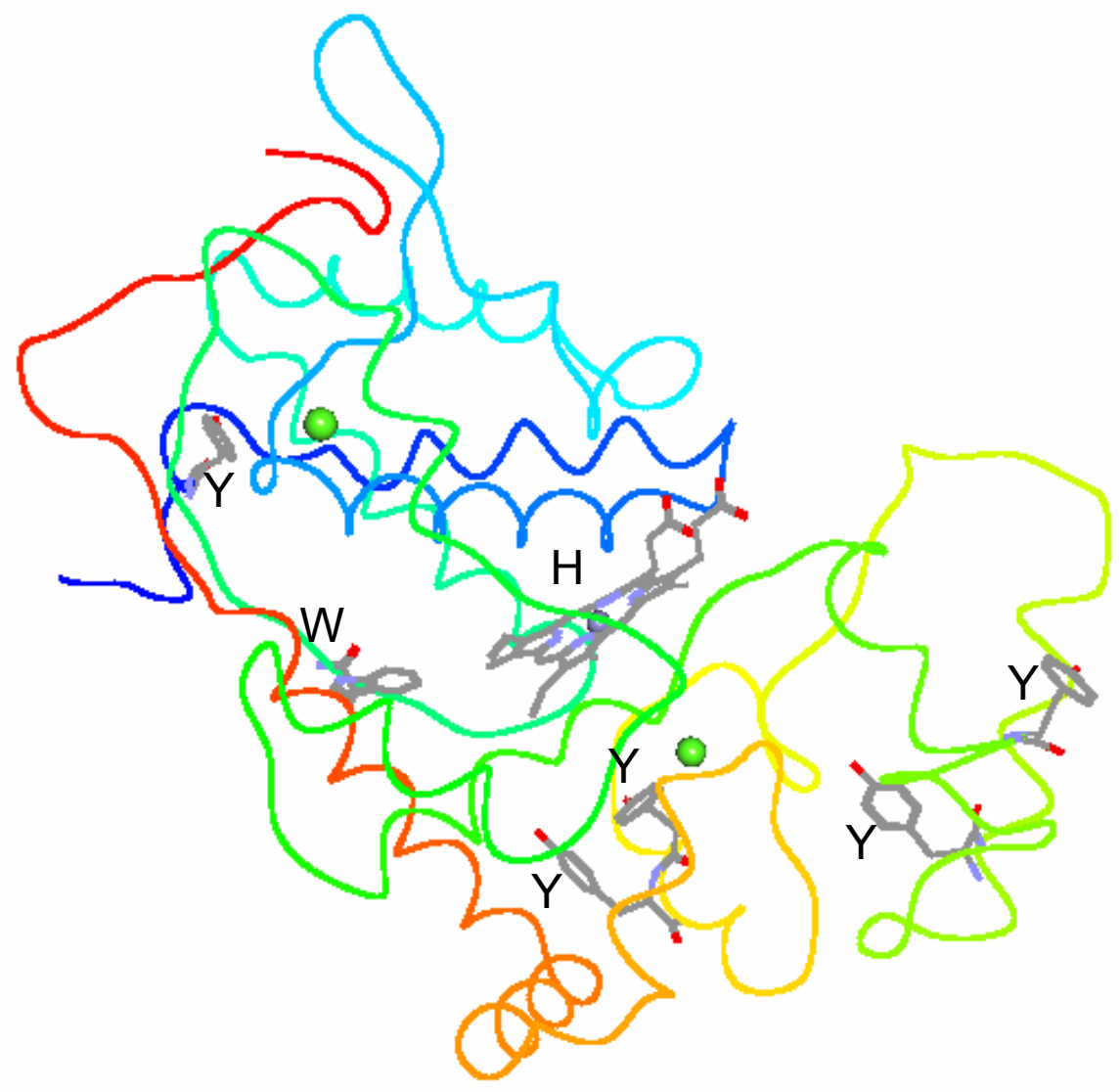

Fig. 1. Cartoon representation of HRP structure showing the relative positions of the tyrosine (Y-7,Y-185, Y-201,Y-233,Y-234), tryptophan $(\mathrm{W}-117)$ and haem $(\mathrm{H})$, as well as associated calcium atoms (green spheres). 


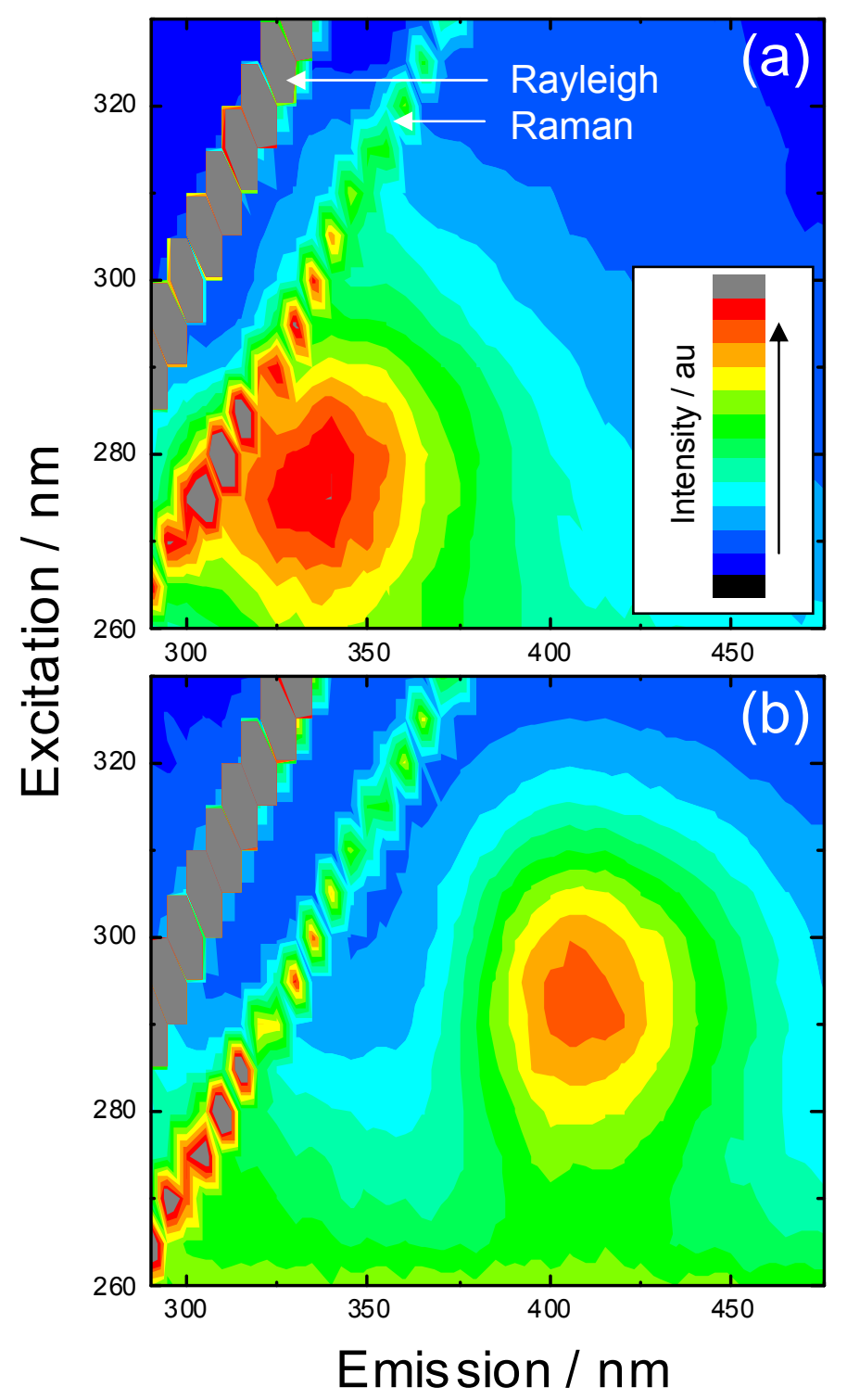

Fig. 2. EEMs of (a) untreated and (b) ultrasound $(378 \mathrm{kHz}$ for 60 minutes) treated HRP solution. 


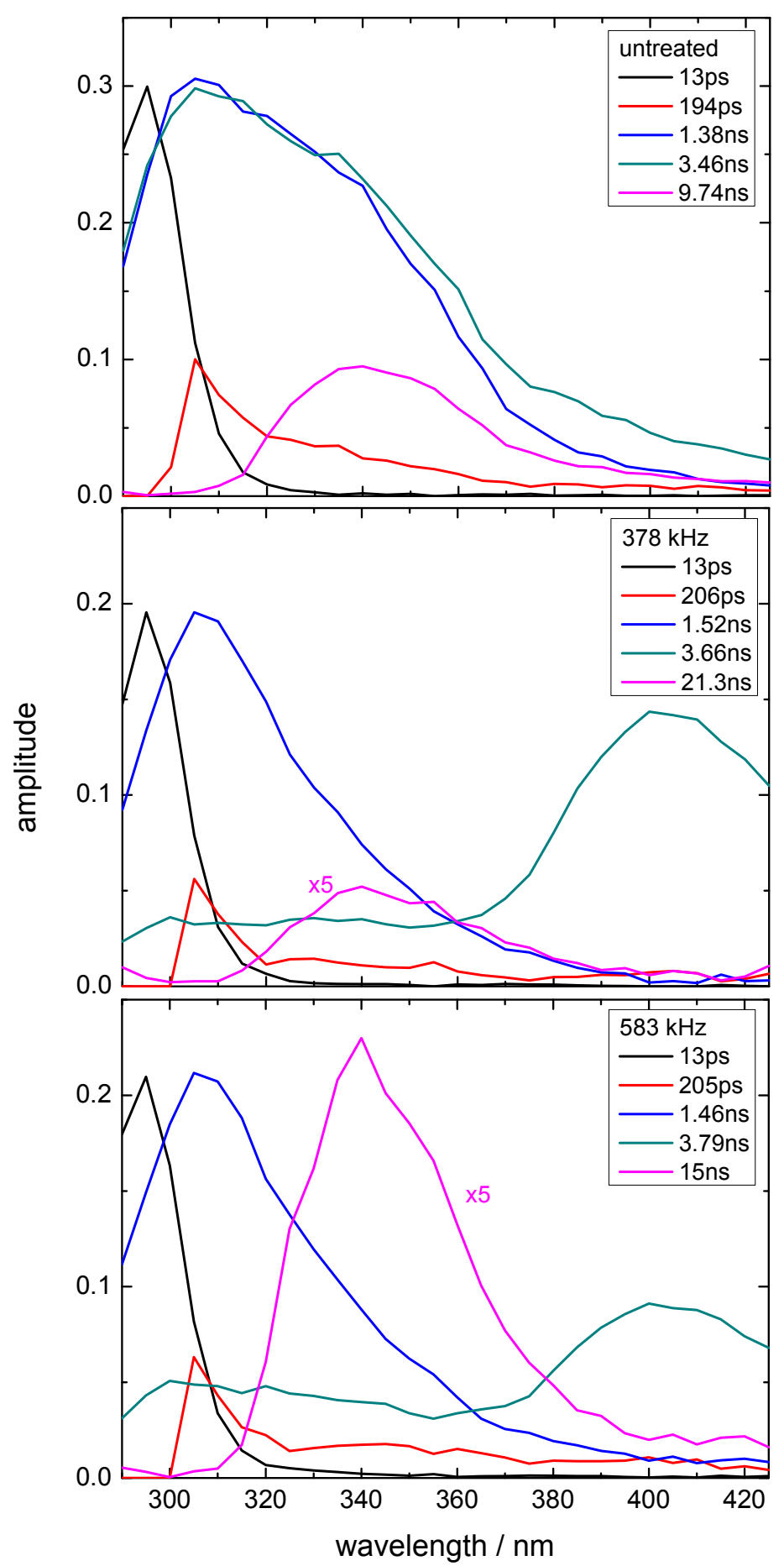

Fig. 3. Decay associated spectra for HRP in solution with and without ultrasound treatment $(378 \mathrm{kHz}$ and $583 \mathrm{kHz}$ for 60 minutes). The short-lived (fixed at 13ps) emission is ascribed to Raman scattering and is shown normalised to the next most intense emission. Other spectral intensities are all relative unless indicated otherwise. The excitation wavelength was $272 \mathrm{~nm}$. 


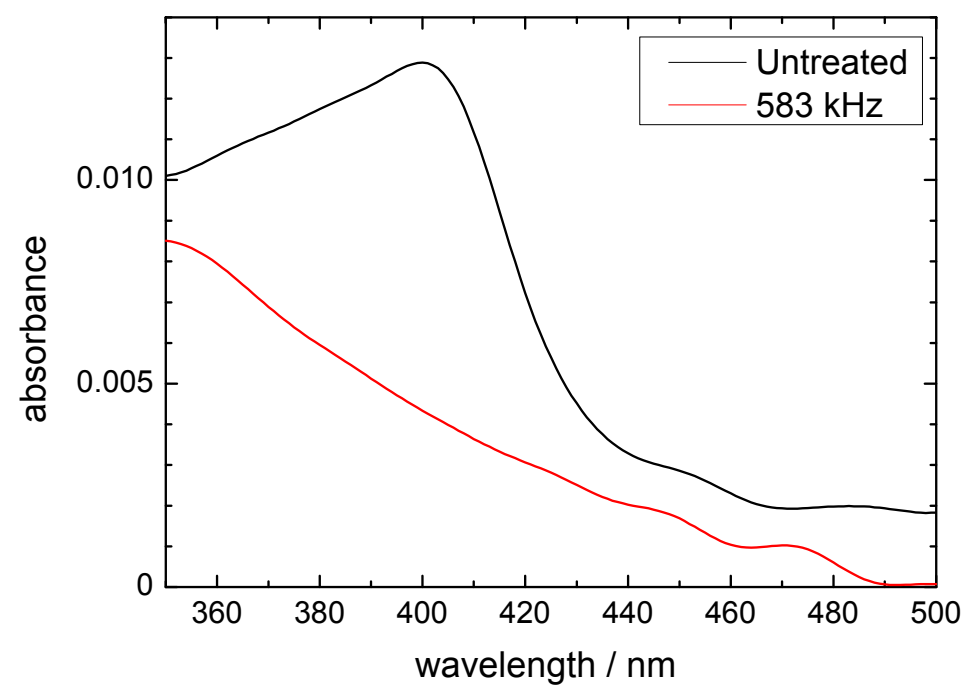

Fig. 4. Absorption spectra, in the haem region, for the untreated HRP sample and that having undergone ultrasound treat at $583 \mathrm{kHz}$ for 60 minutes. 


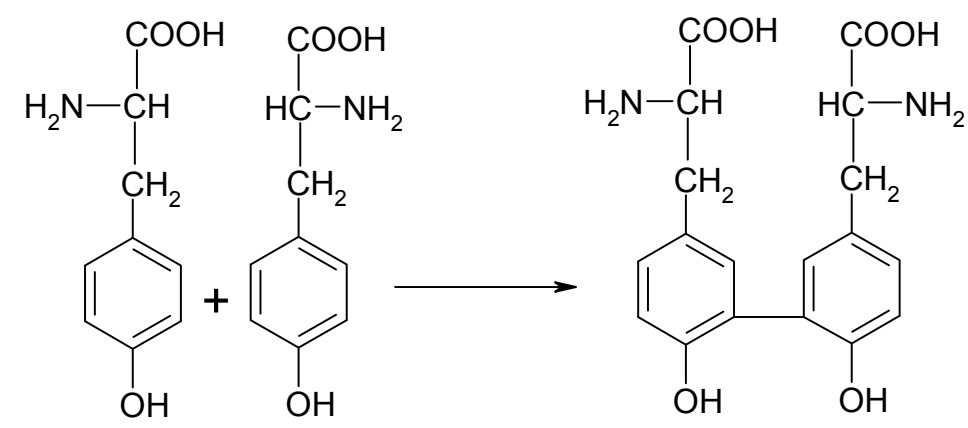

